\title{
AVALLAÇOO DE DOR E DEPRESSÃO EM MULHERES COM ENDOMETRIOSE APÓS INTERVENÇÄO MULTIPROFISSIONAL EM GRUPO
}

Carolina lorençatto, Maria José Navarro Vieira, Andréa Marques, Cristina laguna Benetti-Pinto, Carlos Alberto Petta*

Trabalho realizado no Ambulatório de Endometriose do Centro de Atenção Integral a Saúde da Mulher (CAISM), Universidade Estadual de Campinas, Campinas - SP / Brasil.

*Correspondência Caixa Postal 6181

Cep: $13084-971$

Campinas - Brasil

cpetta@attglobal.net

\section{RESUMO}

OB|ETTV. Avaliar os escores de dor e depressão antes e depois da intervenção multiprofissional em grupo em mulheres com endometriose, bem como verificar as diferenças entre aquelas que não participaram dessa atividade.

Métodos. Participaram deste estudo 128 mulheres do Ambulatório de Endometriose do CAISM/UNICAMP, distribuídas igualmente em dois grupos: que recebeu ou não a intervenção em grupo. Todas preencheram o Inventário de Depressão de Beck e a Escala Visual Analógica.

Resultados. Foi identificada diminuição significativa dos escores de dor e depressão ao final da intervenção em grupo. As mulheres que não participaram dos grupos de apoio mostraram escores maiores de dor quando comparadas aos escores iniciais das participantes dos grupos. Já os escores de depressão foram menores no grupo sem intervenção. Foi identificada correlação positiva entre dor e depressão em ambos os grupos.

Conclusão. A intervenção em grupo foi eficaz na diminuição da dor e depressão das mulheres com endometriose, podendo ser incorporada ao tratamento convencional oferecido às portadoras dessa doença.

Unitermos: Endometriose. Dor pélvica crônica. Depressão. Grupo de apoio.

\section{INTRODUÇÃO}

Atualmente, o tratamento da endometriose tem como principal objetivo o alívio das queixas e dos sintomas, de acordo com as necessidades individuais das mulheres. Em geral, busca-se o alívio da dor pélvica crônica e a recuperação da fertilidade, por meio de medicação e/ou cirurgia. Apesar de todo conhecimento atual sobre a endometriose, ainda não existe uma conduta ideal sem recidiva de sintomas e da doença. Muitas mulheres mantêm a queixa de dor ou de perda da fertilidade mesmo após se submeterem a diversas intervenções, gerando assim, um desgaste emocional devido ao longo caminho percorrido entre o início dos sintomas, diagnóstico e várias tentativas de tratamento da endometriose ${ }^{1-5}$.

Além do sofrimento físico causado pelos sintomas, a endometriose provoca um impacto negativo na vida da mulher, alterando seu rendimento profissional, sua relação familiar e afetiva, reduzindo sua qualidade de vida e principalmente sua auto-estima ${ }^{1,4-9}$.

A relação entre a endometriose e a depressão já foi identificada, sendo que níveis mais elevados são encontrados em mulheres com dor crônica ${ }^{8,9}$.

Devido à diversidade de queixas, atualmente busca-se um tratamento multiprofissional que atenda às demandas físicas e psicológicas das mulheres com endometriose. Há um consenso entre ginecologistas e demais profissionais de saúde sobre a necessidade de suporte psicológico para estas mulheres, porém há poucos relatos que descrevem a realização e os resultados desse tipo de atendimento ${ }^{1-9}$.

Buscando preencher essa lacuna, foi estabelecido, no Ambulatório de Endometriose do CAISM/UNICAMP, um atendimento em grupo envolvendo médicos, psicólogas e fisioterapeutas, nomeado de Grupo de Apoio Psicológico e Fisioterapêutico às Mulheres com Endometriose (GAPFAME). O presente estudo teve como objetivo avaliar os escores de dor e depressão antes e depois do GAPFAME, bem como verificar se há diferenças entre mulheres que não participaram do grupo de apoio.

\section{Métodos}

Participaram deste estudo 128 mulheres do Ambulatório de Endometriose, Departamento de Tocoginecologia da Universidade Estadual de Campinas (UNICAMP), em Campinas (SP). As mulheres foram divididas igualmente em dois grupos: grupo de apoio (participantes do GAPFAME) e grupo sem intervenção (mulheres que não participaram do grupo de apoio).

Ao serem incluídas no Ambulatório de Endometriose, todas as mulheres receberam, na primeira consulta, informações sobre rotinas médicas, exames, procedimentos e atendimentos oferecidos por este serviço. Foram distribuídos folhetos explicativos sobre a doença, os sintomas e também sobre o GAPFAME. Esta intervenção foi oferecida a todas as mulheres na primeira consulta ou no seu retorno médico. Foram encaminhadas ao grupo aquelas que manifestaram desejo de participar, tendo sido realizada inscrição prévia pela equipe de psicologia e, posteriormente, a convocação por carta enviada pelo correio nas semanas que antecederam o início dos grupos. Foram observados, entre as mulheres que aceitaram participar, relatos de sofrimento emocional acentuado e dificuldades nos relacionamentos afetivos e sociais, devido a cronicidade da doença e dos sintomas. 
LOREnÇATTO C ET AL.

Entre as mulheres que não se interessaram em participar, foram relatados, como principais motivos, o trabalho e/ou estudo, além de dificuldades referentes à distância e ao transporte, visto que neste serviço são atendidas pessoas de diversos municípios do Estado de São Paulo e de outros Estados. Também foi observado pelas psicólogas, que algumas mulheres apresentaram resistência ao grupo pelo pensamento de que a equipe de profissionais não estaria acreditando em sua dor, atribuindo sua origem e intensidade ao fator emocional.

Foram analisados e selecionados registros psicológicos e prontuários médicos de mulheres que participaram de dez grupos (realizados semestralmente), de março de 2001 a outubro de 2005. Nesse período, 170 mulheres foram inscritas e convocadas, sendo que apenas 113 compareceram ao início dos grupos. Destas, 74 concluíram e 39 perderam seguimento por motivos variados (desinteresse, alteração na condição financeira, dificuldade de transporte, horário de trabalho, cirurgia, entre outros ignorados).

Das mulheres que concluíram o grupo, 64 foram incluídas neste estudo de acordo com os seguintes critérios: diagnóstico cirúrgico de endometriose, queixa de dor pélvica crônica no início do grupo, ter participado de no mínimo sete encontros, ter preenchido por completo as avaliações clínicas e psicológicas e ter preenchido o registro diário da dor. Foram excluídas aquelas que haviam participado mais de uma vez do GAPFAME.

O grupo que não recebeu intervenção foi selecionado e entrevistado no Ambulatório de Endometriose. Participaram da triagem 98 mulheres, no período de junho a dezembro de 2005, sendo incluídas neste grupo as 64 primeiras que preencheram o critério de inclusão: diagnóstico cirúrgico de endometriose, queixa de dor pélvica crônica, presença de dor no dia da entrevista, não ter participado do grupo de apoio e não ter recebido algum tipo de intervenção psicológica nos últimos seis meses da entrevista. As mulheres foram avaliadas em uma única entrevista, no dia da consulta, ou no retorno médico no ambulatório.

O projeto de pesquisa deste trabalho foi avaliado e aprovado pela Comissão de Pesquisa do Departamento de Tocoginecologia do CAISM e pelo Comitê de Ética em Pesquisa da Faculdade de Ciências Médicas da UNICAMP. As mulheres incluídas no grupo, sem intervenção, assinaram um termo de consentimento livre e esclarecido, pois a coleta de dados foi prospectiva. Devido a análise dos dados do grupo de apoio ser retrospectiva, pois o mesmo faz parte da rotina do serviço do Ambulatório de Endometriose, não se utilizou o termo de consentimento, sendo respeitado o sigilo das fontes de dados.

\section{Materiais avaliativos}

As participantes dos dois grupos preencheram questionário com dados referentes a aspectos sociodemográficos, condição clínica da endometriose e do sintoma de dor, o Inventário de Depressão de Beck $(B D I)^{10}$ e a Escala Visual Analógica (EVA)". As mulheres do grupo de apoio preencheram o registro diário da dor durante o período de intervenção: um instrumento formulado com a finalidade de obter a média semanal da dor, calculada a partir da soma dos escores diários, dividido pelo número de dias registrados. Ao final do grupo, o BDI foi novamente aplicado.

\section{Descrição da intervenção em grupo de apoio}

O GAPFAME é realizado desde março de 200 l e é oferecido a todas as mulheres do Ambulatório de Endometriose do CAISM/ UNICAMP como tratamento complementar aos demais atendimentos e procedimentos usuais (clínico e cirúrgico). Foi definido e estruturado com base nos princípios da terapia cognitiva comportamental e também nas experiências relatadas de outras intervenções multidisciplinares de dor crônica, tendo como principal objetivo transmitir informações sobre a endometriose e promover a reabilitação do bem-estar físico, emocional e social das mulheres com a doença $a^{12-15}$. Foi estruturado em dez encontros semanais, com duração de 2h30, sendo a primeira hora destinada à intervenção fisioterapêutica e, as demais, à intervenção psicológica. $\bigcirc$ número de participantes variou de acordo com as convocações e presenças, sendo o mínimo de quatro e o máximo de 12 mulheres.

Foram objetivos da intervenção fisioterapêutica, promover maior harmonização postural e aumento do metabolismo na região pélvica. Para isso, foram utilizados exercícios isométricos e isotônicos que visavam atuar nos encurtamentos musculares e nas retrações provocadas pela posição antálgica. Os encontros buscavam também elevar a liberação de beta-endorfinas, por meio de exercícios de deslocamentos e atividades lúdicas que proporcionavam clima de descontração e relaxamento, sempre associando música e movimento. Na etapa final, foram propostos alguns exercícios respiratórios e técnicas de mentalização ${ }^{16}$.

A intervenção psicológica seguiu um cronograma estruturado (porém não uniforme) em temas definidos em: autoconhecimento - correlação entre corpo e mente, aceitação, significado e enfrentamento da doença e dor, estresse - teoria e manejo dos sintomas, endometriose - conceitos atuais e tratamentos disponíveis, e sexualidade. Também foram realizadas discussões sobre relacionamentos afetivos (familiares e sociais) e estratégias de resolução de problemas. Em um dos encontros realizados, o médico responsável pelo Ambulatório de Endometriose esteve presente, respondendo e esclarecendo dúvidas sobre a doença.

Os temas foram abordados através de técnicas cognitivas comportamentais, atividades lúdicas e ocupacionais e materiais psicoeducativos, como leituras complementares sobre o tema abordado.

O tamanho amostral foi calculado com base em dois estudos de grupos de intervenção com mulheres que tinham dor crônica. Para o cálculo referente à dor, com uma diferença aceitável entre as médias do escore inicial e final da dor igual a 1,0 e $\alpha=0,05$ e $\beta=0,20$, obteve-se tamanho amostral de 63 mulheres $^{17}$. Para o cálculo de depressão, com diferença aceitável entre as médias do escore inicial e final de depressão igual a 6,0 e $\alpha=0,05$ e $\beta=0,20$, obteve-se um tamanho amostral de 61 mulheres $^{18}$. Desta forma, o tamanho amostral foi definido a partir desses cálculos em 64 mulheres, para ambos os grupos. 


\begin{tabular}{|c|c|c|c|c|c|}
\hline \multirow[b]{2}{*}{ Características } & \multicolumn{2}{|c|}{ Grupo de apoio } & \multicolumn{2}{|c|}{ Gruposem intervenção } & \multirow{2}{*}{$\mathbf{P}$} \\
\hline & $n$ & $\%$ & $\mathrm{n}$ & $\%$ & \\
\hline $\begin{array}{l}\text { Cor } \\
\text { Branca } \\
\text { Negra } \\
\text { Parda/mulata } \\
\text { Outra }\end{array}$ & $\begin{array}{c}37 \\
6 \\
20 \\
1\end{array}$ & $\begin{array}{c}57,8 \\
9,4 \\
31,3 \\
1,6\end{array}$ & $\begin{array}{c}40 \\
4 \\
18 \\
2\end{array}$ & $\begin{array}{c}62,5 \\
6,3 \\
28,1 \\
3,1\end{array}$ & 0,79 \\
\hline $\begin{array}{l}\text { Estado civil } \\
\text { Com união } \\
\text { Sem união }\end{array}$ & $\begin{array}{l}52 \\
12\end{array}$ & $\begin{array}{l}81,3 \\
18,8\end{array}$ & $\begin{array}{l}47 \\
17\end{array}$ & $\begin{array}{l}73,4 \\
26,6\end{array}$ & 0,40 \\
\hline $\begin{array}{l}\text { Filhos } \\
\text { Nenhum } \\
\text { I a } 2 \text { filhos } \\
\text { 3a } 5 \text { filhos }\end{array}$ & $\begin{array}{l}20 \\
32 \\
12\end{array}$ & $\begin{array}{l}31,3 \\
50,0 \\
18,8\end{array}$ & $\begin{array}{l}36 \\
21 \\
7\end{array}$ & $\begin{array}{l}56,3 \\
32,8 \\
10,9\end{array}$ & \\
\hline $\begin{array}{l}\text { Escolaridade } \\
\text { Ensino fundamental } \\
\text { Ensino médio } \\
\text { Ensino superior }\end{array}$ & $\begin{array}{c}29 \\
26 \\
9\end{array}$ & $\begin{array}{l}45,3 \\
40,6 \\
|4,|\end{array}$ & $\begin{array}{l}27 \\
26 \\
11\end{array}$ & $\begin{array}{l}42,2 \\
40,6 \\
17,2\end{array}$ & \\
\hline $\begin{array}{l}\text { Atividade } \\
\text { Não remunerada } \\
\text { Remunerada }\end{array}$ & $\begin{array}{l}18 \\
46\end{array}$ & $\begin{array}{l}28,1 \\
71,9\end{array}$ & $\begin{array}{l}24 \\
40\end{array}$ & $\begin{array}{l}37,5 \\
62,5\end{array}$ & \\
\hline
\end{tabular}

*Teste de Qui Quadrado; \#Teste exato de Fisher.

De acordo com a validação do instrumento para a população brasileira, os pontos de cortes utilizados para a correção do BDI foram: 0 a || = sem depressão ou depressão mínima; de 12 a 19 = depressão leve; de 20 a 35 = depressão moderada e de 36 a $63=$ depressão grave $^{10}$.

\section{Análise estatística}

As associações entre os grupos com e sem intervenção com as características sociodemográficas, grau de endometriose e infertilidade foram avaliadas através dos testes de Qui Quadrado ou exato de Fisher. Para avaliar a EVA entre os grupos foi utilizado o teste de Mann-Whitney. A evolução da EVA durante o tempo da intervenção e a comparação entre momentos do grupo foram avaliadas através de ANOVA, com medidas repetidas de Friedman, seguida de teste de Wilcoxon pareado.

A correlação entre EVA e BDI foi avaliada pelo coeficiente de correlação de Spearman. A associação entre EVA e características sociodemográficas foi avaliada pelo teste de Kruskal-Wallis, seguido de Mann-Whitney. O software utilizado foi o SAS versão $8.2(\mathrm{a}=0.05)$.

\section{Resultados}

As mulheres que participaram do grupo de apoio tinham em

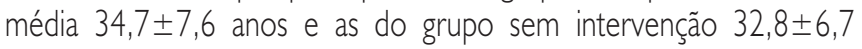
anos. Demais características sociodemográficas, de ambos os grupos, são apresentadas na Tabela I. Diferenças foram encontra-

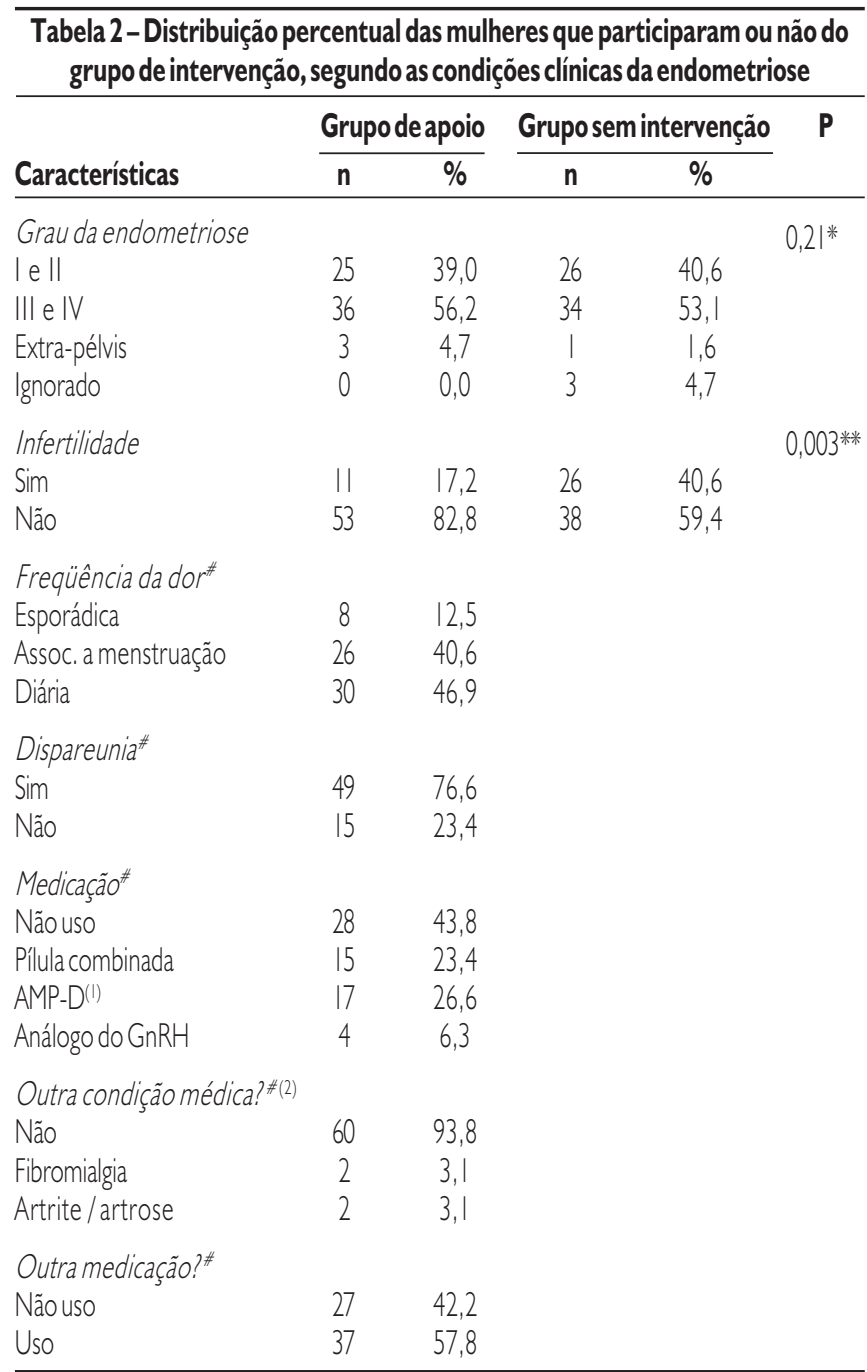

*Teste exato de Fisher, ${ }^{*}$ TTeste de Qui quadrado; " Características avaliadas apenas no grupo de apoio; (1)Acetato de medroxiprogesterona de depósito (AMP-D); (2) Outra condição médica que gerasse 0 sintomadoloroso.

das entre os grupos apenas no que se referiu ao número de filhos: sendo 56\% sem filhos, 33\% de um a dois filhos e $11 \%$ de três a cinco filhos no grupo sem intervenção, e $31 \%$ sem filhos, $50 \%$ de um a dois filhos e 19\% de três a cinco filhos no grupo de apoio $(p=0,0 \mid 7)$.

O tempo médio do diagnóstico da endometriose foi de $36,0 \pm 40,5$ meses no grupo de apoio e de $41,0 \pm 40,1$ meses no grupo sem intervenção $(p=0,58)$. O tempo médio do sintoma doloroso foi de $98,3 \pm 77,5$ meses no grupo de apoio e de $92,6 \pm 83,5$ meses no grupo sem intervenção $(p=0,43)$, e o tempo médio da infertilidade foi de $58,0 \pm 45$, I meses no grupo de apoio e de 66,4 441,5 meses no grupo sem intervenção $(p=0,5 \mathrm{I})$. Não foram encontradas diferenças significativas entre os grupos referentes a essas variáveis.

Quanto ao grau da endometriose, de acordo com o relatório da última cirurgia realizada, utilizando a classificação da American 
LORENÇATTO C ET AL.

Society for Reprodutive Medicine em 1996 19, 39\% das mulheres do grupo de apoio apresentavam grau I e II, 56\% grau III e IV e 5\% extrapélvis. No grupo sem intervenção, $41 \%$ apresentavam grau | e II, 53\% grau III e IV, 2\% extrapélvis e 5\% ignorado. Não foi identificada diferença significativa entre os grupos referente ao grau da endometriose $(p=0,21)$.

A queixa de infertilidade esteve presente em 17\% das mulheres do grupo de apoio e em 59\% do grupo sem intervenção, o que mostra diferença significativa entre os grupos $(p=0,003)$ (Tabela 2).

Algumas características de condição clínica da endometriose foram verificadas apenas no grupo de apoio, descritas na Tabela 2.

A EVA média inicial do grupo de apoio foi $4,2 \pm 3,3$, e do grupo sem intervenção $6,6 \pm 2,4(p=0,0002)$. A EVA avaliada semanalmente no grupo de apoio, a partir dos registros diários da dor, mostrou-se significativamente menor ao longo do tempo da intervenção (da primeira a nona semana) $(p<0,000 \mathrm{l})$, conforme mostra a Tabela 3.

Esta redução também foi identificada quando comparado a EVA entre os momentos do grupo: inicial $x 5^{\mathrm{a}}$ semana $(p<0,000 \mathrm{I})$, inicial $\times 9^{a}$ semana $($ final $)(p=0,0007)$ e $5^{a}$ semana $\times 9^{a}$ semana (final) $(p<0,000 \mathrm{I})$ (Tabela 3).

Quanto à depressão, o escore médio inicial do grupo de apoio foi de 22,8 $\pm 10,2$ e final de 17,0 $\pm 10,2(p<0,0001)$. Para 0 grupo sem intervenção, o escore médio foi de 17,5 $\pm 9,9$, significativamente menor quando comparado ao escore médio inicial do grupo que recebeu a intervenção $(p=0,0049)$ (Tabela 4).

Foi identificada correlação positiva entre EVA e BDI em ambos os grupos, quanto maior o valor da EVA maior o escore do BDI $(p<0,05)$.

No que se referiu à freqüência da dor, foi observado maior escore de EVA entre aquelas com dor diária $(6,2 \pm 2,5)$, do que associada à menstruação $(3,0 \pm 3,2)$ e esporádica $(0,6 \pm 1,1)$ $(p<0,000 \mathrm{I})$. Não foi identificada outra associação significativa entre as características sociodemográficas e demais condições clínicas da endometriose com os escores de EVA e BDI.

\section{Discussão}

As mulheres avaliadas neste trabalho apresentaram condições sociodemográficas semelhantes às descritas em literatura nacional sobre aspectos da epidemiologia da endometriose $e^{3,7,20}$. Diferenças entre os grupos analisados só foram encontradas no que se referiu ao número de filhos e à queixa de infertilidade.

Apesar do longo tempo da dor em ambos os grupos, não foi observada associação significativa dessa variável e das demais avaliadas. Apesar de alguns relatos sobre a relação significativa entre o uso de medicamentos hormonais e a depressão, especialmente os análogos do $\mathrm{GnRH}^{21}$, esta associação não foi observada no estudo.

A frequêencia da dor, relatada pelas mulheres que participaram do grupo de apoio, esteve significativamente associada à EVA. Não foram encontrados estudos com relatos semelhantes de tal associação, significativos ou não. Contudo, este achado já era esperado ao correlacionar a freqüência com a intensidade da dor, visto que

\begin{tabular}{|c|c|c|c|c|c|}
\hline & \multicolumn{2}{|c|}{ Grupo de apoio } & \multicolumn{2}{|c|}{ Grupo sem intervenção } & \multirow[t]{2}{*}{$\mathbf{P}$} \\
\hline & $\mathrm{n}$ & Média(士DP) & $\mathrm{n}$ & Média(士DP) & \\
\hline 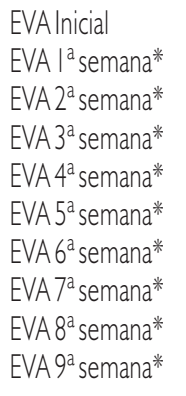 & $\begin{array}{l}64 \\
60 \\
61 \\
63 \\
53 \\
55 \\
55 \\
48 \\
50 \\
51\end{array}$ & $\begin{array}{l}4,2 \pm 3,3 \\
3,9 \pm 2,5 \\
3,8 \pm 2,7 \\
3,6 \pm 2,7 \\
3,2 \pm 2,7 \\
3,2 \pm 2,8 \\
3,3 \pm 2,5 \\
3,2 \pm 2,9 \\
2,6 \pm 2,5 \\
2,6 \pm 2,6\end{array}$ & 64 & $6,6 \pm 2,4$ & $0,0002^{\#}$ \\
\hline \multicolumn{5}{|c|}{$\begin{array}{l}\text { Comparação de mudança da EVA ao longo do } \\
\text { tempo do grupo }\end{array}$} & $<0,\left.000\right|^{(1)}$ \\
\hline \multicolumn{5}{|c|}{$\begin{array}{l}\text { Comparação entre os momentos do grupo } \\
\text { EVA Inicial x EVA } 5^{\mathrm{a}} \text { semana* } \\
\text { EVA Inicial x EVA } 9^{\mathrm{a}} \text { semana* } \\
\text { EVA } 5^{\mathrm{a}} \text { semana* } \text { EVA }^{\mathrm{a}} \text { semana* }\end{array}$} & $\begin{array}{l}<0,\left.000\right|^{(2)} \\
0,0007^{(2)} \\
<0,\left.000\right|^{(2)}\end{array}$ \\
\hline
\end{tabular}

*EVA referenteàmédiasemanal de dor obtida através do registro diário da dor preenchida apenas pelas mulheres do grupo de apoio;

\#Teste de Mann-Whitney: comparação EVAinicial: grupo de apoio x grupo sem intervenção;

(I)Análise de variância de Friedman;

(2) Teste de Wilcoxon pareado.

\begin{tabular}{|c|c|c|c|c|c|}
\hline \multicolumn{6}{|c|}{$\begin{array}{l}\text { Tabela 4 - Distribuição e comparação da média dos escores do BDI } \\
\text { das mulheres que participaram ou não do grupo de apoio, segundo } \\
\text { os momentos de avaliação }\end{array}$} \\
\hline & \multicolumn{2}{|c|}{ Grupo de apoio } & \multicolumn{2}{|c|}{ Grupo sem intervenção } & \multirow[t]{2}{*}{$\mathbf{P}$} \\
\hline & $n$ & Média(士DP) & $\mathrm{n}$ & Média $( \pm D P)$ & \\
\hline $\begin{array}{l}\text { BDl inicial } \\
\text { BDI final }\end{array}$ & $\begin{array}{l}64 \\
64\end{array}$ & $\begin{array}{l}22,8 \pm 10,2 \\
17,0 \pm 10,2\end{array}$ & 64 & $17,5 \pm 9,9$ & $0,0049^{\prime \prime}$ \\
\hline
\end{tabular}

Comparação BDI entre momentos do grupo

BDl inicial XBDI final $\quad<0,\left.000\right|^{* *}$

*Teste de Mann-Whitney: comparação BD linicial: grupo de apoio x grupo sem intervenção; * * Teste deWilcoxon pareado.

a pessoa com dor diária apresenta-se mais queixosa e com maior probabilidade de exacerbar o relato da dor do que aquela com dor esporádica ${ }^{22,23}$.

A comparação entre os grupos - que receberam ou não a intervenção - permitiu observar diferenças significativas entre os escores de dor e depressão nas mulheres avaliadas nesse estudo. A média de dor encontrada no grupo sem intervenção foi maior do que a relatada pelas mulheres no início do grupo de apoio. Em recente estudo realizado em nosso meio, a EVA obtida na préintervenção medicamentosa foi de $7,3 \pm 0.3^{24}$, valor aproximado à média encontrada no grupo que não recebeu a intervenção. Não houve nenhum critério específico na seleção dos grupos que justificasse a diferença encontrada referente à EVA. Sabemos que 
por mais confiável que seja, a mensuração da dor por meio de escalas apresenta limitações, pois este sintoma trata-se de uma manifestação subjetiva multifatorial influenciada pelo estado emocional da pessoa.

Referente à depressão, o grupo sem intervenção apresentou escores mais baixos do que o escore inicial do grupo de apoio. Esta diferença, já esperada, mostrou que as mulheres que aceitaram e/ou procuraram participar do grupo de apoio apresentavam mais sintomas depressivos, isto é, possivelmente sentiam-se em pior estado emocional, mesmo apresentando escores mais baixos de dor, do que aquelas avaliadas no ambulatório que não receberam a intervenção. Dados semelhantes foram descritos quanto ao estresse percebido, concluindo-se que as mulheres que se sentiam piores procuraram auxílio complementar ao tratamento convencional20.

De acordo com a classificação dos escores do BDI proposta para a população brasileira $^{10}$, a média encontrada no grupo sem intervenção corresponde à depressão leve, ao passo que a média inicial do grupo de apoio corresponde à depressão moderada. Em recente pesquisa, foi detectada a presença de depressão em $86 \%$ das mulheres com endometriose e dor pélvica crônica e em 38\% das mulheres sem dor. Referente à distribuição em graus, entre aquelas com dor, 34\% eram leve e $52 \%$ de moderada à grave, não sendo identificada associação entre a dor e o grau da depressão?.

As análises realizadas entre os momentos do grupo de apoio, referente à EVA e ao BDI, mostraram melhora das mulheres, com redução significativa dos escores de dor e depressão. Tanto a comparação de mudança ao longo do tempo, como a comparação entre os momentos (inicial, quinta e nona semana) foram significativas. O mesmo aconteceu com a depressão, significativamente menor no final do grupo. Dados que se assemelham aos descritos em literatura, referentes aos grupos de intervenção multiprofissional para a dor crônica ${ }^{12,13,15,17,18}$.

Embora a diminuição da intensidade da dor tenha sido um evento significativo, o alívio total não ocorreu na maioria das participantes, o que já era esperado. A proposta do grupo de apoio não é extinguir o sintoma doloroso, visto que o tratamento da dor na endometriose envolve também o uso de medicações hormonais e intervenções cirúrgicas. Porém, a redução na intensidade da dor pode representar melhora no bem-estar físico e emocional destas mulheres, uma vez que as torna menos incapacitadas para a realização de suas tarefas e funções, recuperando parte da vitalidade perdida com a persistência da dor. Ao final dos grupos, freqüentemente observavam-se relatos de melhoras referentes ao aspecto físico e emocional associados à diminuição da dor. A intervenção psicológica e fisioterapêutica, proposta no grupo de apoio, foi delineada justamente com este objetivo: melhorar a qualidade de vida das mulheres com endometriose.

A presença da depressão no final da intervenção pode ser devido ao fato de que algumas queixas dolorosas são semelhantes às queixas depressivas, o que pode exacerbar o diagnóstico da depressão em pessoas com dor crônica. $\mathrm{Na}$ escala do BDI, são descritos alguns itens como fadiga, distúrbios do sono, inibição para o trabalho, diminuição da libido, preocupação somática, entre outros, comuns no relato da pessoa com dor, sem que necessaria- mente esteja com depressão. Alguns autores sugerem que o ponto de corte do BDI seja no mínimo 20 pontos para população do contexto médico, para evitar a superestimação dos sintomas depressivos ${ }^{25}$. Neste caso, o escore médio final não seria considerado como presença de depressão.

A relação entre endometriose e depressão já foi demonstrada?, sendo que, neste estudo, escores mais elevados da dor estiveram diretamente associados à intensidade da depressão. Esta associação já foi identificada anteriormente, sendo que alguns autores apontam a depressão como uma conseqüência direta da dor, porém não há consenso nesta questão temporal ao definir qual condição precede a outra ${ }^{14,26}$. É possível afirmar, no entanto, que as duas condições coexistem e que uma agrava a experiência da outra ${ }^{14}$.

O impacto de uma doença crônica, como a endometriose, associado ao sintoma doloroso persistente traz mudanças significativas; inicialmente ocorre a perda de um corpo saudável e ativo, que pode gerar um estado de dependência e limitações. Comportamentos inadequados e ganhos secundários à dor se instalam e a pessoa apresenta tendência de isolamento e/ou de se sentir vítima. Os relacionamentos, no geral, ficam prejudicados, pois geralmente as pessoas em volta se cansam de tantas queixas. É comum o relato de que são taxadas como "Maria das dores". As mudanças provocadas pela queda do desempenho físico, muitas vezes levam estas mulheres a perdas no fator econômico. As percepções cognitivas alteradas pelo estresse crônico provocado pela doença geram, na maioria dos casos, sentimentos de desvalia e incapacidade, favorecendo então a reação depressiva.

- modelo cognitivo da depressão explica esse transtorno psicológico como resultante de interpretações distorcidas dos diversos acontecimentos da vida, determinadas pelos esquemas (padrões cognitivos) formulados diferentemente em cada pessoa, com base em suas experiências anteriores. Em grande parte, as distorções cognitivas geram uma visão negativa de si, do mundo e do futuro, que formam o que se denominou de tríade cognitiva da depressão. A partir daí, instala-se o círculo vicioso da depressão, em que a pessoa tende a, cada vez mais, interpretar todas as suas experiências como negativas, produzindo os chamados pensamentos negativos e autodestrutivos, que são freqüentemente observados em portadores de dor crônica ${ }^{23,27}$.

A maioria das intervenções multiprofissionais em pessoas com dor crônica, de diversas origens, baseia-se nestes conceitos cognitivistas e comportamentais; que já se mostraram eficazes na diminuição da dor e da depressão ${ }^{12,13,15,17,18}$.

Apesar de não proporcionar alívio total das queixas de dor e depressão, o modelo utilizado no grupo de apoio às mulheres com endometriose também se mostrou eficaz, concordando com os relatos descritos anteriormente.

\section{ConClusão}

Foi verificado que as mulheres que buscaram o grupo de ajuda sentiam-se piores emocionalmente, isto é, apresentavam escores mais elevados de depressão.

Os resultados deste estudo confirmam a necessidade de atendimento multiprofissional, que ofereça suporte psicológico para as 
LORENÇATTO C ET AL.

mulheres com endometriose e dor pélvica crônica. $O$ grupo de apoio realizado mostrou ser um bom aliado no tratamento convencional, uma vez que proporcionou redução nos escores de dor e depressão.

Conflito de interesse: não há.

\section{SUMMARY}

Evaluation of pain and DEPRESSION IN WOMEN WITH ENDOMETRIOSIS AFTER A MULTI- PROFESSIONAL GROUP INTERVENTION

OBIECTIVE. Evaluate pain and depression scores before and after multi-professional group intervention in women with endometriosis as compared to those who did not participate in this activity.

Methods. A total of 128 women attended at the Endometriosis Outpatient Facility CAISM/UNICAMP, was assessed and distributed equally into two groups: women who received group intervention and those who did not. All women completed the Beck Depression Inventory and the Analogical Visual Scale.

RESULTS. There was a significant decrease in pain and depression scores at the end of group intervention. Women who had not participated in the intervention had higher initial scores of pain when compared to those who had. On the other hand, in the group without intervention, depression scores were lower. A positive correlation was identified between pain and depression in both groups.

Conclusion. Group intervention was efficient in diminishing pain and depression in women with endometriosis and may be included in the conventional treatment for patients affected by this disease. [Rev Assoc Med Bras 2007; 53(5): 433-8]

KeY wORDS: Endometriosis. Chronic pelvic pain. Depression. Support group.

\section{REFERÊNCIAS}

I. Damario MA, Rock JA. Pain recurrence: a quality of life issue in endometriosis. Int I Gynaecol Obstet. 1995;50:S27-42.

2. Valle RF, Sciarra JJ. Endometriosis: treatment strategies. Ann N Y Acad Sci. 2003;997:229-39.

3. Arruda MS, Petta CA, Abrão MS, Benetti-Pinto CL. Time elapsed from onset of symptoms to diagnosis of endometriosis in a cohort study of Brazilian women. Hum Reprod. 2003;18:756-9.

4. Cox H, Henderson L, Andrersen N, Cagliarini G, Ski C. Focus group study of endometriosis: Struggle, loss and the medical merry-go-round. Int J Nurs Pract. 2003;9:2-9.

5. Denny E. Women's experience of endometriosis. J Adv Nurs. 2004;46:64I-8.

6. Christian, A. The relationship between women's symptoms of endometriosis and self-esteem. J Obstet Gynecol Neonatal Nurs. 1993;22:370-6.

7. Marques A, Bahamondes L, Aldrighi JM, Petta CA. Quality of life in Brazilian women with endometriosis assessed through a medical outcome questionnaire. J Reprod Med. 2004;49: I I 5-20.
8. Waller KG, Shaw RW. Endometriosis, pelvic pain, and psychological functioning. Fertil Steril. 1995;63:796-800

9. Lorençatto C, Petta CA, Navarro MJ. Bahamondes L, Matos A Depression in women with endometriosis with and without chronic pelvic pain. Acta Obstet Gynecol Scand. 2006;85:88-92.

I 0. Cunha JA. Manual da versão em português das Escalas Beck. São Paulo: Casa do Psicólogo; 2001

| I. Huskisson E. Measurement of pain. Lancet. |974;2: | |27-3 I.

12. Turk DC, Meichenbaum DH. A cognitive-behavioural approach to pain management. In: Wall PD, Melzack R. Textbook of pain. 2nd ed. London: Churchill Lingstone; 1989. p.I00I-9.

I 3. Flor H, Fydrich T, Turk DC.Efficacy of multidisciplinary pain treatment centers: a meta-analytic review. Pain. 1992;49:221-30.

I 4. Rosenthal RH. Psychology of chronic pelvic pain. Obstet Gynecol Clin North Am. 1993;20:627-42.

15. Wood DP, Wiesner MG, Reiter RC. Psychogenic chronic pelvic pain: diagnosis and management. Clin Obstet Gynecol. 1990;33:179-95.

16. Metzger D. An integrated approach to the management of endometriosis. In: Steege J, Metzger D, Levy B. Chronic pelvic pain an integrated approach. Philadelphia: Saynders Company; 1998. p.12634.

17. Albert H. Psychosomatic group treatment helps women with chronic pelvic pain. J Psychosom Obstet Gynecol. 1999;20:216-25.

18. Kames LD, Rapkin AJ, Naliboff BD, Afifi S, Ferrer-Brechner T. Effectiveness of an interdisciplinary pain management program for the treatment of chronic pelvic pain. Pain. 1990;41:4I-6.

19. American Society For Reproductive Medicine. Revised American Society for Reproductive Medicine classification of endometriosis: 1996. Fertil Steril. |997;67:817-21.

20. Petrelluzzi KFS. Dor, estresse e qualidade de vida em mulheres com endometriose:avaliação de um protocolo de intervenção [dissertação]. Campinas: Universidade Estadual de Campinas; 2005.

21. Warnock JK, Bundren JC, Morris DW. Sertraline in the treatment of depression associated with gonadotropin-releasing hormone agonist therapy. Biol Psychol. 1998;43:464-5.

22. Oliveira JT de. Aspectos comportamentais das síndromes de dor crônica. Arq Neuro-Psiquiatr. 2000:58:360-5.

23. Vandenberghe L. Abordagens comportamentais para a dor crônica. Psicol Reflex Crit. 2005; | 8:47-54

24. Petta CA, Ferriani RA, Abrão MS, Hassan D, Rosa E Silva JC, Podgaec $S$, et al. Randomized clinical trial of a levonorgestrel-releasing intrauterine system and a depot $\mathrm{GnRH}$ analogue for the treatment of chronic pelvic pain in women with endometriosis. Hum Reprod. 2005;20: 1993-8

25. Kalichman SC, Sikkema KJ, Somlai A. Assessing persons with human immunodeficiency virus (HIV) infection using the Beck Depression Inventory: disease processes and other potential confounds. J Pers Assess. 1995:64:86-100.

26. Fishbain DA, Cutler R, Rosomoff HL, Rosomoff RS. Chronic painassociates depression: antecedent or consequence of chronic pain? a review. Clin J Pain. 1997; |3:| |6-37.

27. Beck AT, Rush AJ, Shaw BF, Emery G. Terapia cognitiva da depressão. Porto Alegre: Ed. Artes Médicas; 1997.

Artigo recebido: 10/4/07

Aceito para publicação: 4//07 